\title{
العلاقة المظهرية والجينية لانواع جنس .Streptomyces spp. المعزولة من الترب الملوثة بالهيدروكاربونات
}

Phenetic and Phylogenetic Analysis of Streptomyces spp. Isolated from Hydrocarbons Polluted Soil

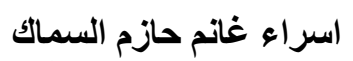

Essra Gh. Alsammak

College of Science/ University of Mosul

Essra_alsammak@yahoo.com

يهذف البحث الى تسليط الضوء على دور التصنيف المتعدد Polyphasic والذي يضم كل من التصنيف المظهري والجيني لتحديد وتثبيت

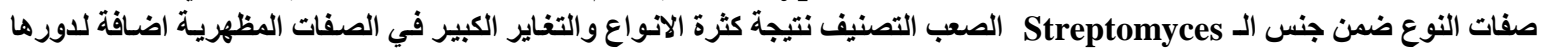

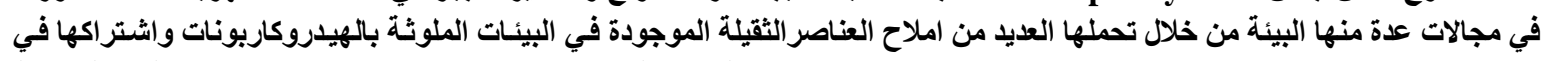

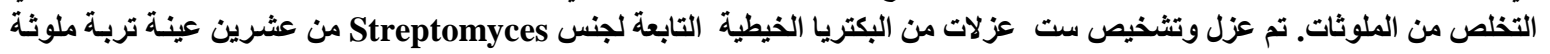

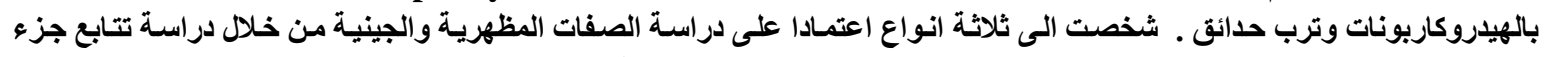

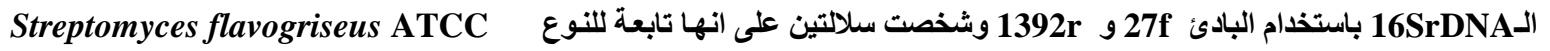

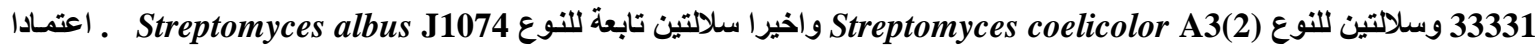

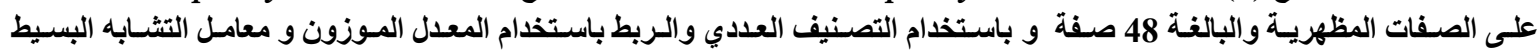
باستخام البرنامج الاحصائي SPSS Simple matching coefficient (Ssm)

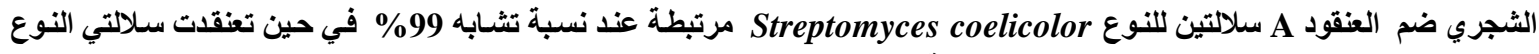

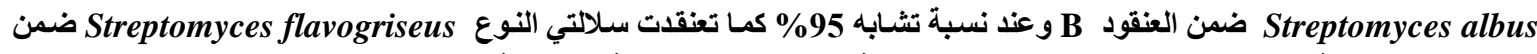

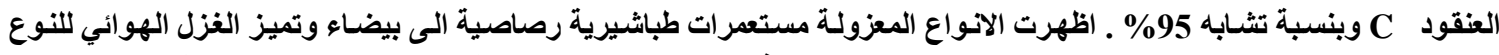

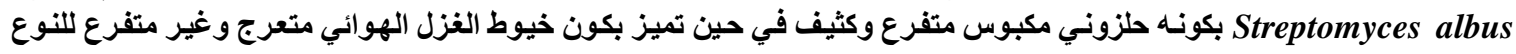
Streptomyces coelicolor متركن Streptomyces flavogriseus

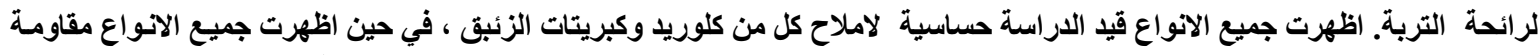

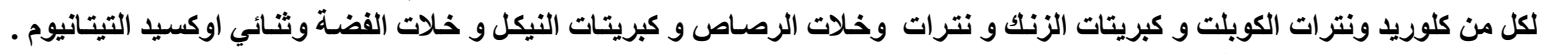

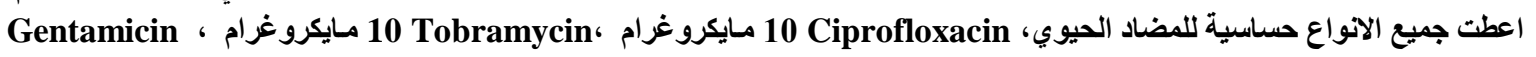

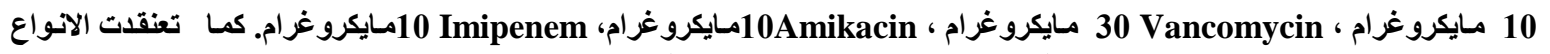

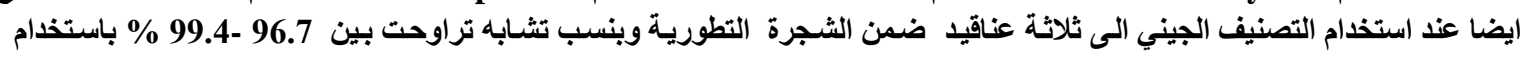
Maximum likelihood method وطنيفة Clustal W

Abstract

$$
\text { الكلمات المفتاحية: 16s rDNA، التصنيف المظهري ، التصنيف الجيني. }
$$

The research aims to highlight the role of polyphasic classification, which includes both phenotypic and genetic classification to determine and stabilize species characteristics within the genus Streptomyces, which is difficult to classify due to the large number of species and the large heterogeneity of phenotypic traits, in addition to its role in several areas, including Environments through resistance to many salts of heavy elements present in environments contaminated with hydrocarbons and their participation in the disposal of pollutants. In this study 6 isolates of Streptomyces obtained from 20 hydrocarbons contaminated and garden soil have been isolated and identified through phenotypic \& genotypic characteristics using nucleotide sequence of the 16s rDNA gene by universal primers $27 \mathrm{f} \& 1392 \mathrm{r}$ following strains were identified to two strains that similar to Streptomyces flavogriseus ATCC 33331 , two strains belongs to Streptomyces albus $\mathbf{J 1 0 7 4} \&$ two strains belongs Streptomyces coelicolor A3(2). Depending on 48 of phenotypic characteristic by using numerical taxonomy, average linkage method and simple matching coefficient (Ssm) using statistical program SPSS. The strains grouped to three clusters in hierarchical tree, the two strains belongs to Streptomyces coelicolor grouped in cluster A at similarity of $99 \%$, the two strains belongs to Streptomyces albus grouped in cluster B at similarity of $95 \%$ \& the two strains that belongs to Streptomyces flavogriseus accumulate in cluster $\mathrm{C}$ at similarity of $95 \%$. The color of colony of all strain was grey to white ,the aerial mycelium of Streptomyces coelicolor was branched spiral \& rectalflex, while Streptomyces albus have branched condense spiral aerial mycelium, Streptomyces 
flavogriseus produced unbranched \& rectalflex aerial mycelium. all of strains produced earthy odor. The all isolates showed tolerant to Nickel sulphate, Zinc sulphate, Lead acetate\& nitrate, Silver acetate, Titanium dioxide, Cobalt nitrate \& chloride. Also all isolates showed sensitivety to Mercuric sulphate $\&$ chloride. The results showed that all of strains were sensitive to Ciprofloxacin10 $\mu$, Gentamicin10 $\mu \mathrm{g}$, Vancomycin $30 \mu \mathrm{g}$, Amikacin $10 \mu \mathrm{g}$, Imipenem $10 \mu \mathrm{g}$, Tobramycin $10 \mu \mathrm{g}$. In phylogenetic tree the species clustered in three group also as in phenetic tree in similarity percent between (96.7- 99.4\%) by Clustal W program and Maximum likelihood method, using Mega5.

Key words: 16s rDNA, phenotypic taxonomy, genotypic taxonomy.

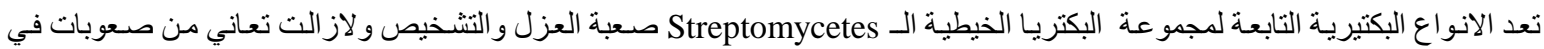

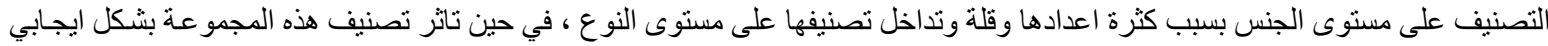

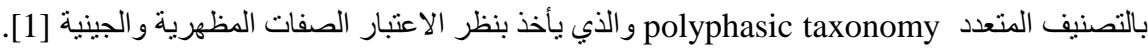

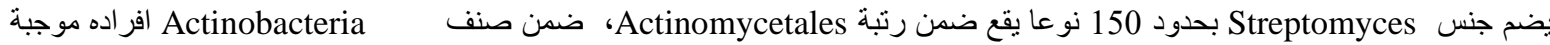

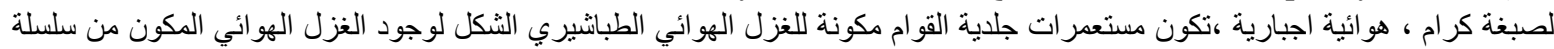

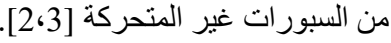

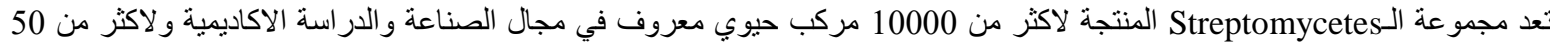

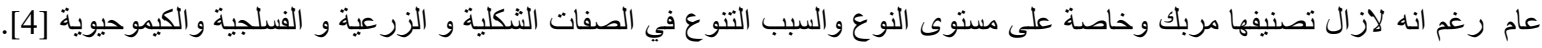

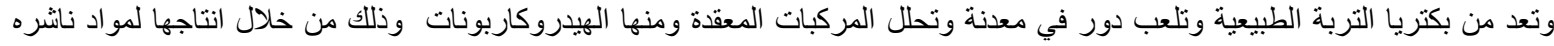

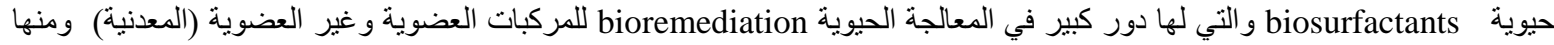
المعادن الثقيلة [6، كـ5].

المواد وطر ائق العمل اخذت 20 عينة تربة من مناطق ملوثة بالهيدروكاربونات البترولية و لا سيما مناطق وجود مخلفات المولدات الكهربائية لمناطق مختلفة من

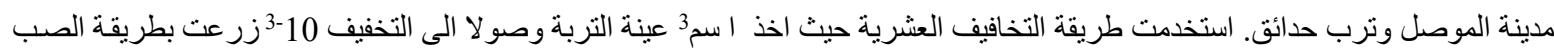

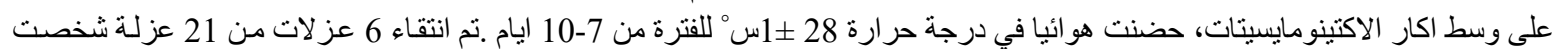

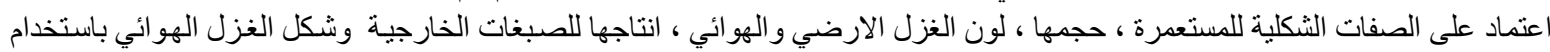

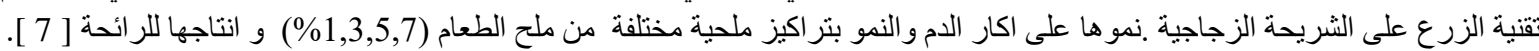
التحمل للمعادن الثقيلة

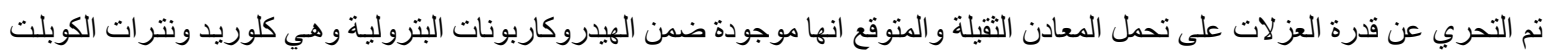

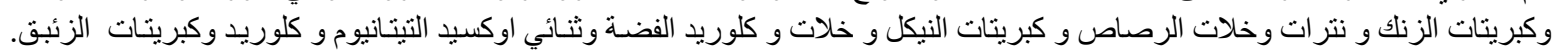

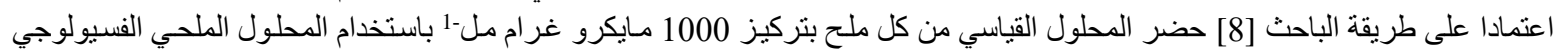

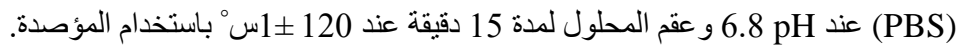

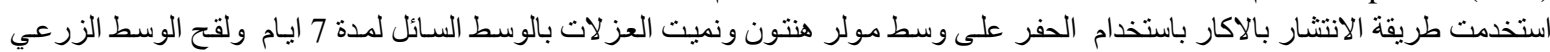

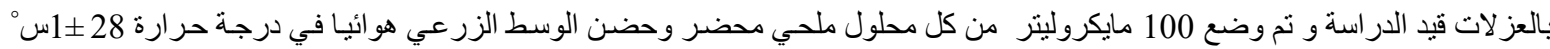

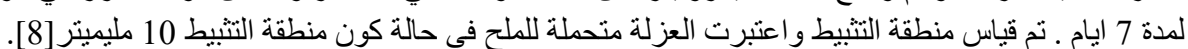
الحساسية للمضادات الحيوية تمناية

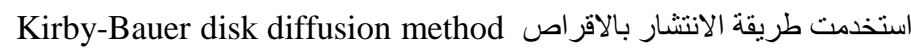
المعتمدة من قبل مل مational Committee for Clinical Laboratory Standards NCCLS [9] باستخدام المضـادات الحيويـة الاتيـة:

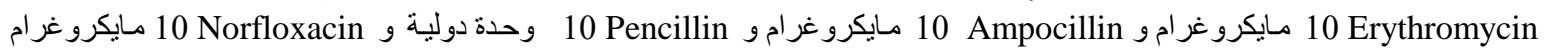

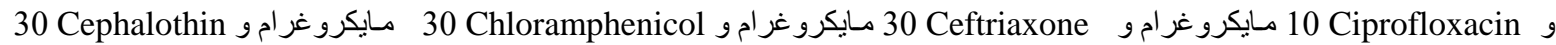

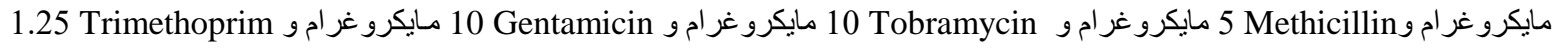

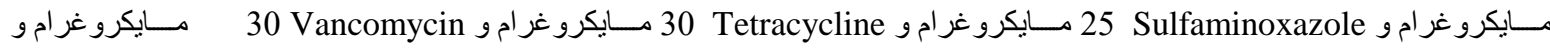

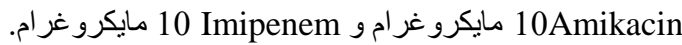

\section{التشخيص الجيني عزل الانا الجرثئومي البئي}

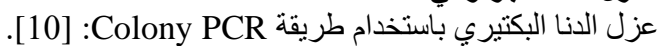

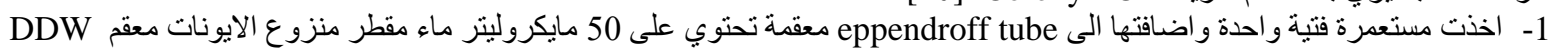

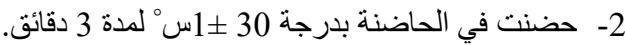

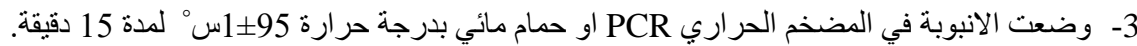

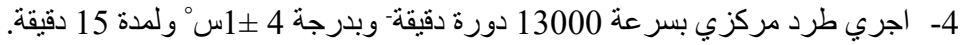


تقدير الانا وقياسه

قدرت نقاوة وتركيز الدنا المستخلص من العينات المعزولة باستخدام جهاز Biodrop Spectrophotometer شركة

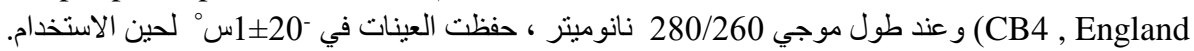

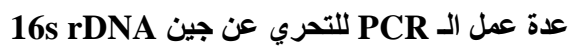

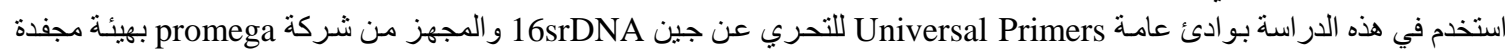
(1) و Lophilized form

\begin{tabular}{|c|c|c|}
\hline أسم الجين المستخدم & $5 \rightarrow 3$ & $ت$ \\
\hline 16srDNA & $\begin{array}{l}\text { AGAGTTTGATCCTGGCTCAG } \\
\text { GACGGGCGGTGTGTAC }\end{array}$ & $\begin{array}{l}\text { 27f } \text { Upstream } \\
\text { 1392r Downstream }\end{array}$ \\
\hline
\end{tabular}

16s rDNA تفاعلات PCR للتحري عن جين

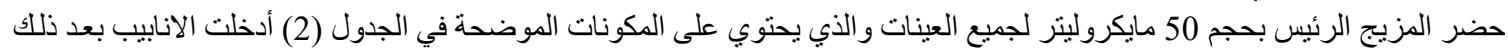

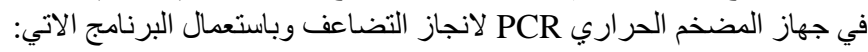

جدول (2): أحجام الإضافات في مزيج تفاعل PCR

\begin{tabular}{|c|c|}
\hline حجم العينة / مايكروليتر & المكونات \\
\hline 14 & D.W \\
\hline 25 & Green master mix \\
\hline 1.5 & Primers forward \\
\hline 1.5 & Primers reverse \\
\hline 8 & DNA template \\
\hline 50 مايكروليتر & حجم العينة النهائي \\
\hline 50 نانوغرام/مايكروليتر & التركيز النهائي \\
\hline
\end{tabular}

\begin{tabular}{lc|c}
$\min$ & 5 & 1 Cycle \\
$\sec$ & 35 & \\
$\min$ & 1.35 & 35 Cycle \\
$\min$ & 1.35 & \\
$\min$ & 10 & 1 Cycle
\end{tabular}

رحلت العينات بجهاز الترحيل الكهربائي ،باستخدام هلام الاكاروز 2\% بفولتية 50 لمدة 75 دقيقة ومن ثم فحص الهام باستخدام الاشعة

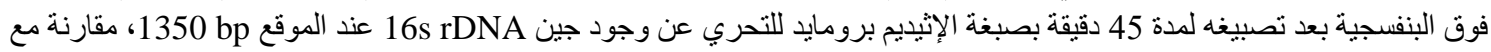
(promega)100bp ladder

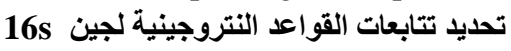

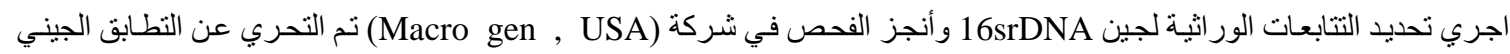
باستخدام برنامج (BLAST) و المنوفر في المركز الوطني لمعلومـات التقانات الحياتية ( http://www.ncbi.nlm.nih.gor) و على الموقع (NCBI) National Center Biotechnology Information 
العلاقة التطورية للسلالات المشخصة

Phylogenetic relationships of strains

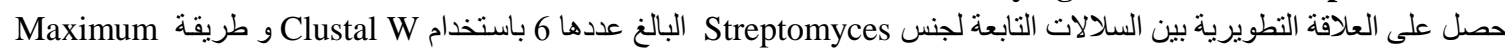
likelihood النتائج والمناقشة شخصت ست عز لات انها تابعة لجنس Streptomyces من الترب الملوثة بالهيدروكاربونات اعتمادا على الصفات المظهريـة ومنها شكل

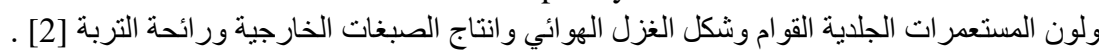

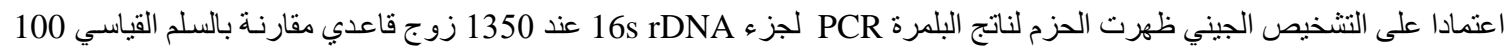

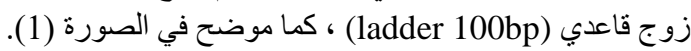

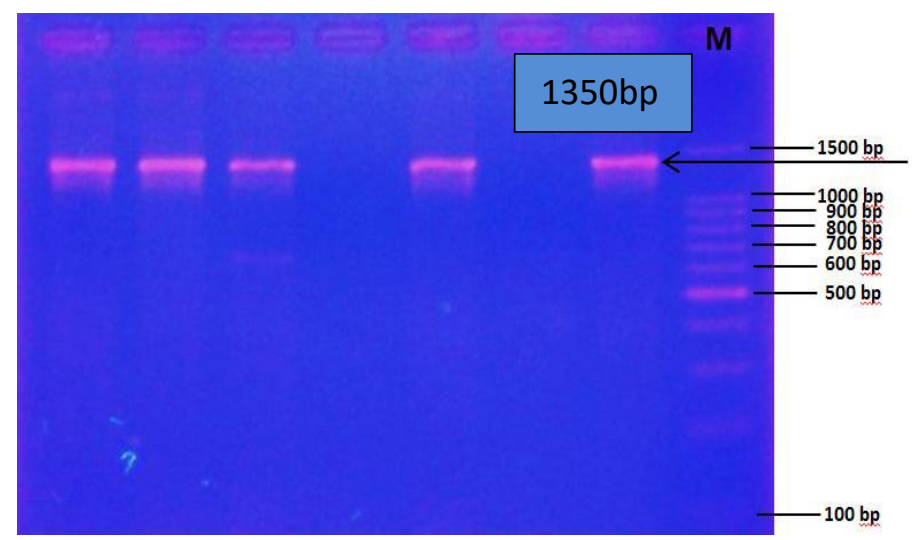

$$
\text { صورة (1): الحزم التابعة لجزء 16s rDNA عند } 1350 \text { زوج قاعدي مقارنة بالسلم القياسي } 100 \text { زوج قاعدي }
$$

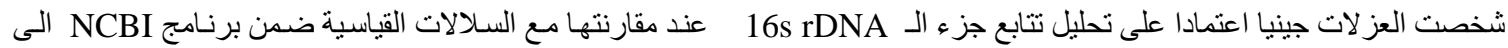

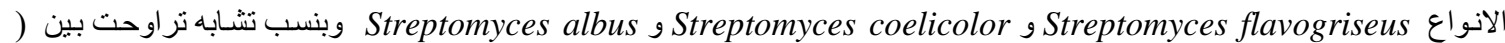
94- 96 \% \% عما موضح في شكل (1) وجدول (3).

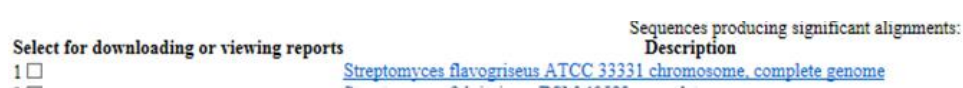
$\begin{array}{ll}\text { Select for downloading or viewing reports } & \text { Sequences producing signiticant alignments: } \\ \text { Description } & \text { Streptomvces flavogriseus ATCC } 33331 \text { chromosome, complete genome }\end{array}$

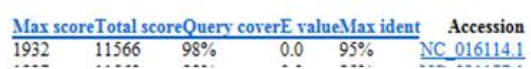

$\frac{\text { Max scoreTotal scoreQuerv corerE valueMax ident }}{1866}$ Accession

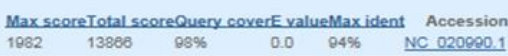

Select for downloading or viewing reports
$1 \square$ $\frac{\text { Max scoreTotal scoreQuery corerE value Max ident }}{1762}$ Accession

Max scoreTotal score Query coverE valueMax ident Accession $1941 \quad 11035 \quad 94 \% \quad 0.998 \%$ MC 003898

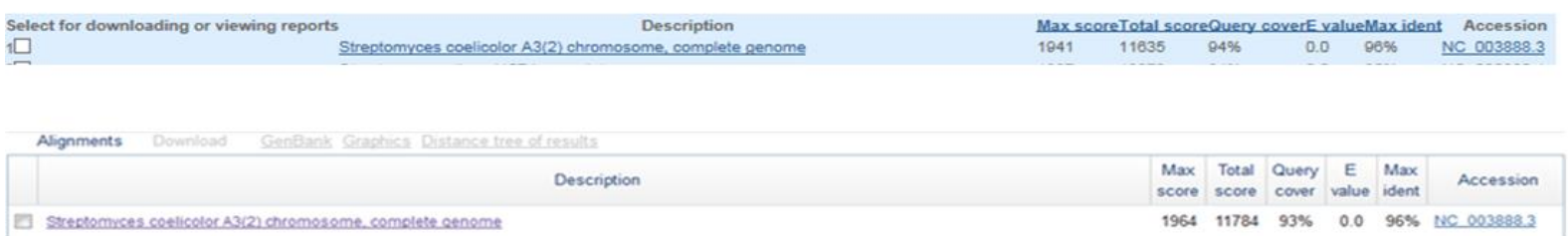

شكل (1): نتائج تثخيص العزلات التابعة لجنس Streptomyces اعتمادا على تحليل تتابع جزء الـ 16s rDNA عند مقارنتها

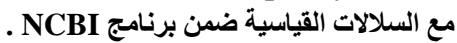




\begin{tabular}{|c|c|c|c|c|}
\hline \multicolumn{2}{|c|}{ مقارنة بالسلالات القياسية ضمن برنامج } & \multirow{2}{*}{ على تحليل تتابع جزء الـ 6s rDNA6 } & \multicolumn{2}{|c|}{ جدول (3): تثخيص الانواع المعزولة اعتمادا } \\
\hline نسبة التشابه مع السلالات القياسية & & & منطقة العزل & $\bar{ت}$ \\
\hline Streptomyces flavogriseus ATCC33331 & $95 \%$ & Streptomyces flavogriseus & تربة ملوثة بالهيدروكاربونات & 1 \\
\hline Streptomyces flavogriseus ATCC33331 & $96 \%$ & Streptomyces flavogriseus & تربة ملوثة بالهيدروكاربونات & 2 \\
\hline Streptomyces albus J1074 & $94 \%$ & Streptomyces albus & تربة ملوثة بالهيدروكاربونات & 3 \\
\hline Streptomyces albus J1074 & $95 \%$ & Streptomyces albus & تربة ملوثة بالهيدروكاربونات & 4 \\
\hline Streptomyces coelicolor A3(2) & $96 \%$ & Streptomyces coelicolor & تربة ملوثة بالهيدروكاربونات & 5 \\
\hline Streptomyces coelicolor $\mathrm{A} 3(2)$ & $96 \%$ & Streptomyces coelicolor & تربة حديقة & 6 \\
\hline
\end{tabular}

أوجدت العلاقة المظهرية بين الانواع المعزولة باستخدام التصنيف العددي وذلك بمقارنة عدد 48 من الصفات الثكلية و الفسلجية باستخدام

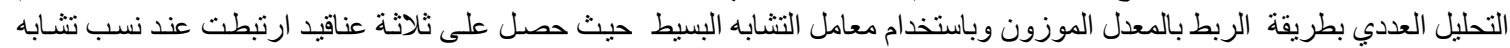

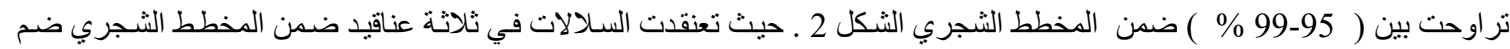

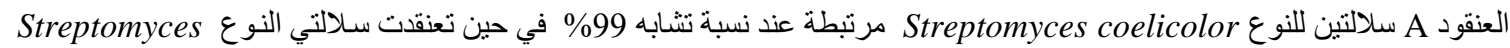

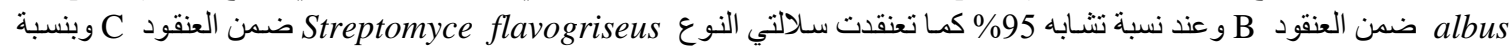
تشابه 95\% .

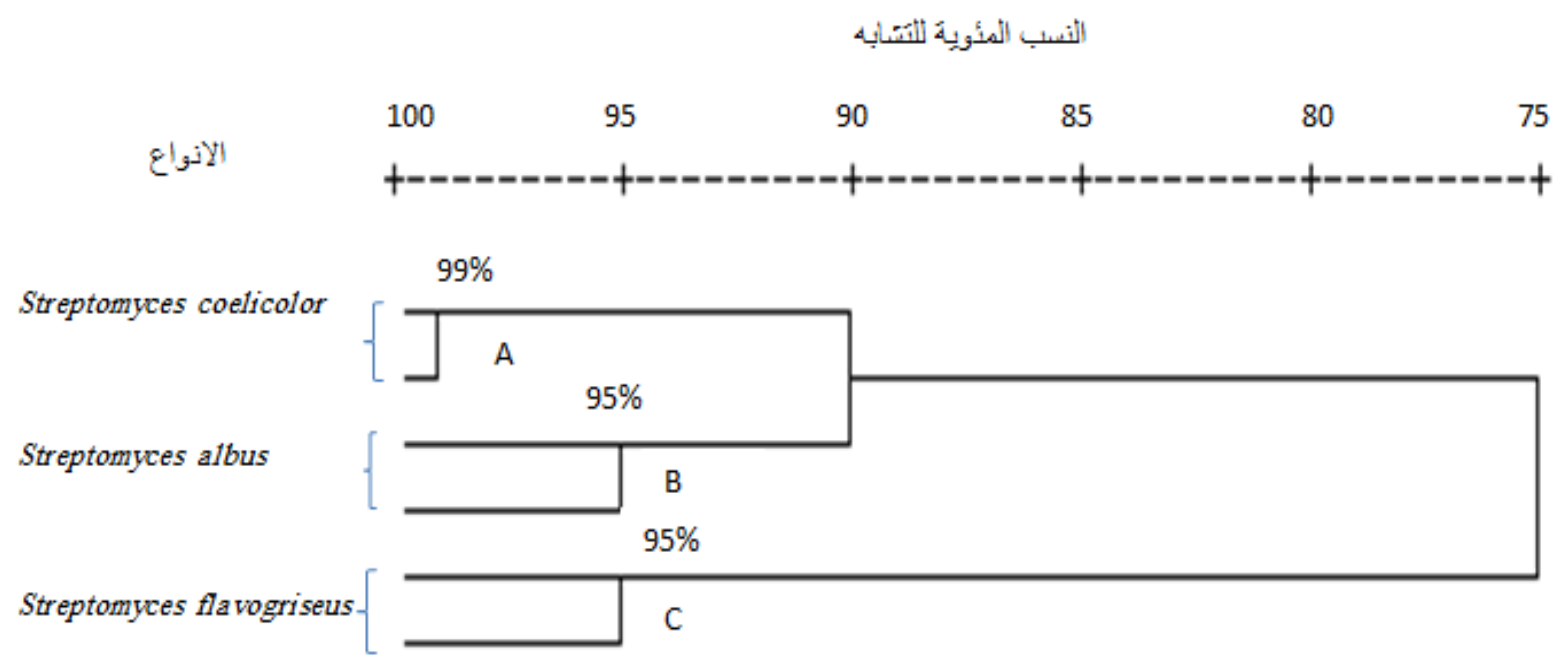

شكل (2): المخطط الثجري للأنواع قيد الدراسة باستخدام الربط بالمعدل الموزون ومعامل التشابه البسيط 
جدول (4 ): النسب المئوية للصفات للانواع التابعة لجنس Streptomyces ضمن العناقيد في الثجرة التصنيفية المظهرية شكل (2)

\begin{tabular}{|c|c|c|c|c|}
\hline $\mathbf{C}$ & B & $\mathbf{A}$ & \multicolumn{2}{|c|}{ رمز العنقود } \\
\hline $\begin{array}{l}\text { Streptomyces } \\
\text { flavogriseus }\end{array}$ & streptomyces albus & $\begin{array}{c}\text { Streptomyces } \\
\text { coelicolor }\end{array}$ & \multicolumn{2}{|c|}{ الانواع } \\
\hline 2 & 2 & 2 & \multirow{2}{*}{\multicolumn{2}{|c|}{ الصفات السلالات }} \\
\hline \multicolumn{3}{|c|}{ النسب المئوية للصفات } & & \\
\hline $\mathbf{m}$ & 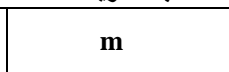 & m & حجم المستعمرة & فوسات المستعمرة على هنتون \\
\hline $\mathbf{0}$ & $\mathbf{0}$ & $\mathbf{0}$ & ملتصقة & |القوام \\
\hline 100 & 0 & 0 & برتقالي & \multirow{3}{*}{ اللون } \\
\hline $\mathbf{0}$ & 100 & $\mathbf{0}$ & عسلي & \\
\hline $\mathbf{0}$ & $\mathbf{0}$ & 100 & قهوائي & \\
\hline 100 & 100 & 100 & طباثيري & سطح المستعمرة \\
\hline 50 & 50 & 50 & ابيض & \multirow{2}{*}{ اللون } \\
\hline 100 & 100 & 100 & رصاصي & \\
\hline $\mathbf{0}$ & 100 & 100 & متفرع & \multirow{2}{*}{ تُكل الغزل الهوائي } \\
\hline $\mathbf{0}$ & 100 & 50 & حلزوني & \\
\hline 100 & $\mathbf{0}$ & 50 & متعزج & \multirow{4}{*}{ |ج الصبغات الخارجية } \\
\hline $\mathbf{0}$ & 100 & 0 & لـزون مكبوس كثيف & \\
\hline 100 & $\mathbf{0}$ & $\mathbf{0}$ & برتقالية او وردية & \\
\hline $\mathbf{0}$ & $\mathbf{0}$ & 0 & قهوائية & \\
\hline 100 & 100 & 100 & \multicolumn{2}{|c|}{ انتاج رائحة التربة مجات } \\
\hline 100 & 0 & 100 & \multicolumn{2}{|c|}{ تحليل الام خلال 24 سـاعة } \\
\hline 100 & $\mathbf{0}$ & 100 & \multicolumn{2}{|c|}{ تحليل الدم خلال 72 سـاعة } \\
\hline 100 & 100 & 100 & \begin{tabular}{l|l}
$\% 1$ \\
\end{tabular} & \multirow{4}{*}{ 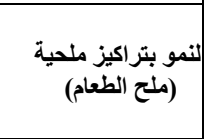 } \\
\hline 100 & 100 & 100 & $\% 3$ & \\
\hline 100 & 100 & 100 & $\% 5$ & \\
\hline $\mathbf{0}$ & 50 & 100 & $\% 7$ & \\
\hline 100 & 100 & 100 & \multicolumn{2}{|c|}{ كلوريد الزئبق } \\
\hline $\mathbf{0}$ & $\mathbf{0}$ & $\mathbf{0}$ & \multicolumn{2}{|c|}{ كلوريد الكويلت } \\
\hline 100 & 100 & 100 & \multicolumn{2}{|c|}{ كبريتات الزئبق } \\
\hline $\mathbf{0}$ & $\mathbf{0}$ & $\mathbf{0}$ & \multicolumn{2}{|c|}{ كبريتات الزنتك } \\
\hline $\mathbf{0}$ & $\mathbf{0}$ & $\mathbf{0}$ & \multicolumn{2}{|c|}{ نترات الكويلت } \\
\hline $\mathbf{0}$ & $\mathbf{0}$ & $\mathbf{0}$ & \multicolumn{2}{|c|}{ نترات الرصاص } \\
\hline $\mathbf{0}$ & $\mathbf{0}$ & $\mathbf{0}$ & \multicolumn{2}{|c|}{ كبريتات النيكل } \\
\hline 0 & $\mathbf{0}$ & $\mathbf{0}$ & \multicolumn{2}{|c|}{ خلات الرصاص } \\
\hline $\mathbf{0}$ & $\mathbf{0}$ & $\mathbf{0}$ & \multicolumn{2}{|c|}{ خلات الفضة } \\
\hline $\mathbf{0}$ & $\mathbf{0}$ & $\mathbf{0}$ & كسيد التيتانيوم & ثنائي او \\
\hline 50 & 50 & $\mathbf{0}$ & ريد الفضة & \\
\hline $\mathbf{0}$ & $\mathbf{0}$ & $\mathbf{0}$ & Ampicillin & $10 \mu \mathrm{g}$ \\
\hline $\mathbf{0}$ & $\mathbf{0}$ & $\mathbf{0}$ & Penicillin & $10 \mathrm{iu}$ \\
\hline $50 \mathrm{~m}$ & 50 & $50 \mathrm{~m}$ & Norfloxaci & $10 \mu \mathrm{g}$ \\
\hline 0 & 50 & 0 & Erythromyc & n $10 \mu \mathrm{g}$ \\
\hline 100 & 100 & 100 & Ciprofloxaci & $\mathrm{n} 10 \mu \mathrm{g}$ \\
\hline $\mathbf{0}$ & $\mathbf{0}$ & $\mathbf{0}$ & Ceftriaxon & $30 \mu \mathrm{g}$ \\
\hline $50 / 50 \mathrm{~m}$ & $100 \mathrm{~m}$ & $\mathbf{0}$ & Chloramphen & $\operatorname{col} 30 \mu \mathrm{g}$ \\
\hline 0 & 0 & 0 & Cephalothi & $130 \mu \mathrm{g}$ \\
\hline $\mathbf{0}$ & $\mathbf{0}$ & 0 & Methicillir & $5 \mu \mathrm{g}$ \\
\hline 100 & 100 & 100 & Tobramyci & $10 \mu \mathrm{g}$ \\
\hline 100 & 100 & 100 & Gentamycir & $10 \mu \mathrm{g}$ \\
\hline $\mathbf{0}$ & $\mathbf{0}$ & 0 & $\begin{array}{l}\text { Trimethoprin } \\
\text { sulfamethaxa }\end{array}$ & $\begin{array}{l}1.25 \mu \mathrm{g} \\
0125 \mu \mathrm{g}\end{array}$ \\
\hline 0 & 50 & $\mathbf{0}$ & Tetracyclin & $30 \mu \mathrm{g}$ \\
\hline 100 & 100 & 100 & Vancomyci & $30 \mu \mathrm{g}$ \\
\hline 100 & 100 & 100 & Amikacin & $10 \mu \mathrm{g}$ \\
\hline 100 & 100 & 100 & Imipenem & $10 \mu \mathrm{g}$ \\
\hline
\end{tabular}

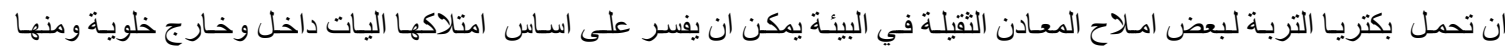

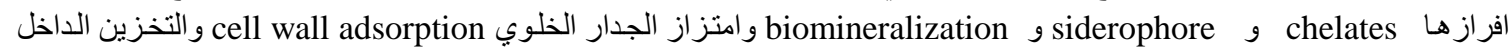

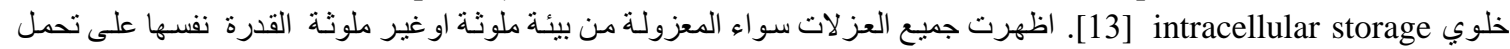

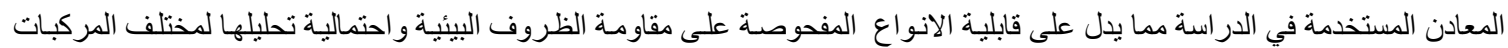




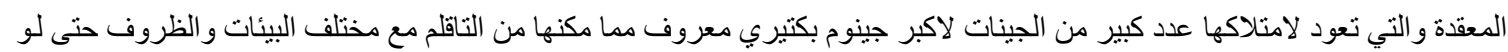

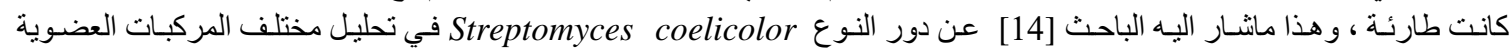

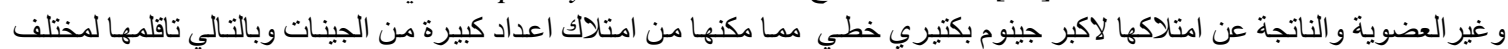
البيئات.

حصل على المخطط التطوري للعلاقة بين الانواع اعتمـادا على تحليل تتابعات جزء الـ16srDNA باستخدام الـ

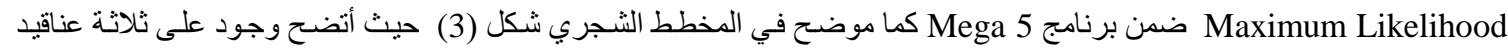

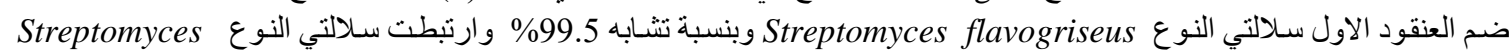

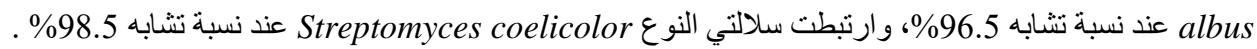

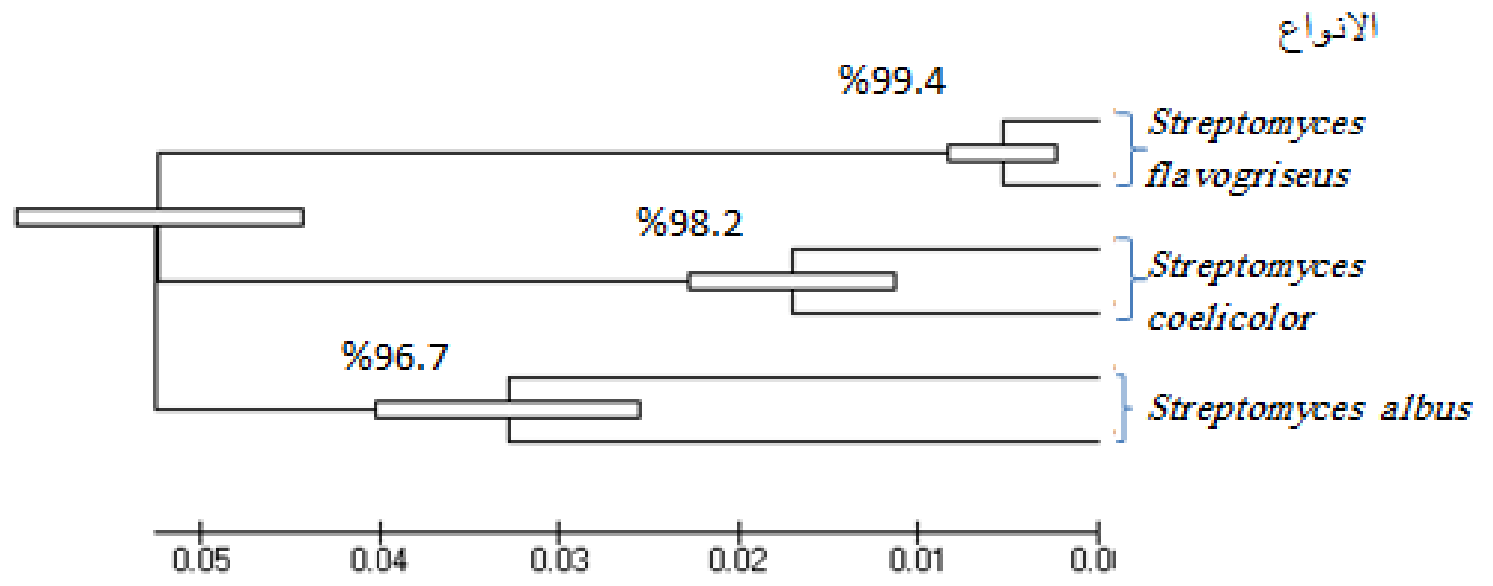

\section{شكل (3): العلاقة الجينية بين الانواع قيد الدراسة اعتمادا على دراسة تتابع جزء الـ16srDNA باستخدام طريقة الـ Clustal WaxA وطريقة Mega ضمن برنامج 5 Maximum Likelihood}

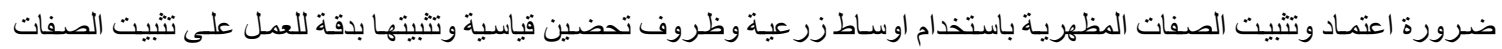

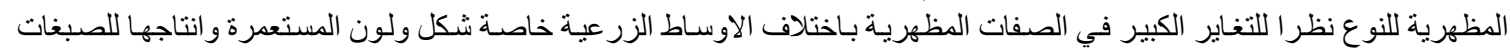

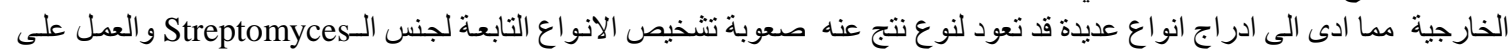

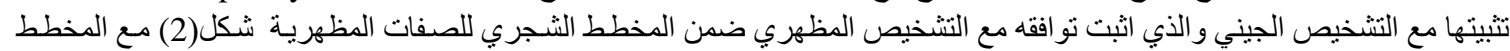

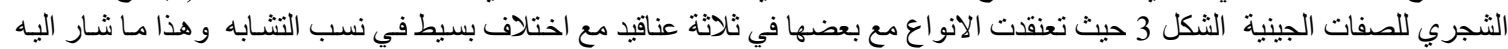

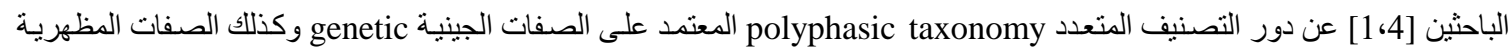

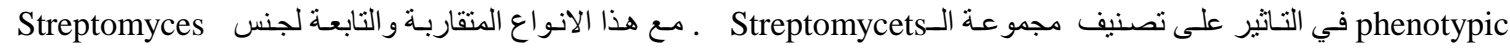

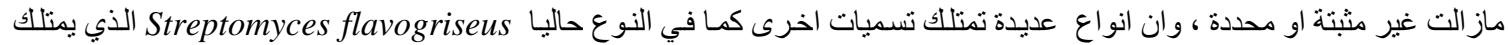

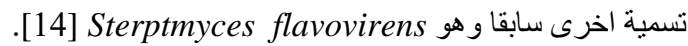

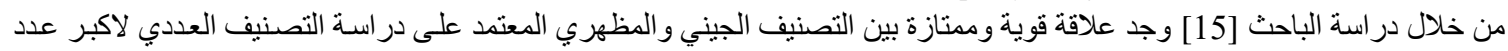

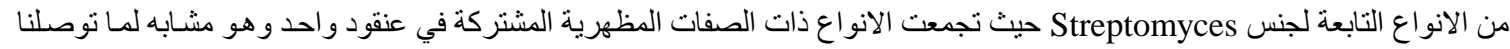

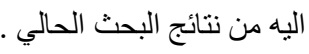

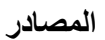

1. Rong, X. and Huang, Y. (2010). Taxonomic evaluation of the Streptomyces griseus clade using multilocus sequence analysis and DNA-DNA hybridization, with proposal to combine 29 species and three subspecies as 11 genomic species. International Journal of Systematic and Evolutionary Microbiology . 60: 696-703.

2. Madigan, T.M., Martinko, M.J., Stahl, A.D. and Clark, P.D. (2013). Brock Biology of Microorganisms. Thirteenth Edition publishing as Benjamin Cummings, San Francisco.

3. Garrity, G.M., Bell, J.A. and Lilburn, T.C. (2004). Taxonomic outline of the Prokaryotes Bergey's Manual of Systematic Bacteriology . $2^{\text {nd }}$ ed. Springer New york, Inc. U.S.A, pp1-401.

4. Anderson, A. S. and Wellington, E.M.H. (2001). The taxonomy of Streptomyces and related genera . International Journal of Systematic and Evolutionary Microbiology. 51:797-814.

5. Chater, K. F. , Biro, S ., Lee, K. J., Palmer, T. and Schrempf, H. (2010).The complex extracellular biology of Streptomyces. FEMS Microbiol Rev . 34:171-198.

6. Karanth, N.G.K., Deo, P. G. and Veenanadig, N. K. (2005). Microbial production of biosurfactants and their importance. http://www.ias.ac.in/currsci/jul10/articles19.htm. 
7. Holt, J. G., Krieg, N. R., Sneath, P.H.A., Staley, J. T., and Williams, S. T. (2000). Bergeys Manual of Determination Bacteriology. $9^{\text {th }}$ ed., Lipponcott Williams and Wilkins, Philadelphia, USA., pp: 189-255.

8. Lakshmipathy, T.D., Prasad, S.A. and Kannabiran, K. (2010). Production of biosurfactant and heavy metal resistance activity of Streptomyces sp.VITDDK3-a novel halo tolerant Actinomycetes isolated from Saltpan Soil . Advances in Biological Research. 4 (2): 108-115.

9. National Committee for Clinical Laboratory. (2011). Performance Standards for Antimicrobial Susceptibility Testing-Twenty-First Information Supplement. NCCLs.M100-21.31(1). National Committee for Clinical Laboratory Clinical and Laboratory Standards Institute. USA.

10. Bodour, A.A., Drees, K.P. and Maier, R.M. (2003). Distribution of biosurfactant-producing bacteria in undisturbed and contaminated Arid Southwestern Soils. Appl. Environ. Microbiol. 69( 6): 3280-3287.

11. Lane, D. J. (1991). 16S/23S rRNA sequencing. in nucleic acid techniques in bacterial systematics. Stackebrandt E., Goodfellow M.John Wiley \& Sons, Chichester, United Kingdom. pp. 115-175.

12. Tamura, K. and Nei, M. (1993). Estimation of the number of nucleotide substitutions in the control region of mitochondrial DNA in humans and chimpanzees. Molecular Biology and Evolution. 10:512-526.

13. Kothe, E., Dimkpa, Ch., Haferburg, G., Schmidt, A., Schmidt, A . and Chutze, E. (2010). Soil Biology :soil heavy metals. Vol 19 . springer Berlin Heidelberg. pp.225-235.

14. Bently, S.D.,Chater, K.F., Cerdeno-Tarraga, A.M., Challis, G.L., Thomson, N.R., James, K.D. et al. (2002). Complete genome sequence of the model actinomycetes Streptomyces coelicolor A3(2). Nature.417:141-147. online

15. Labeda, D.P., Goodfellow, M., Brown, R., Ward, A.C., Lanoot, B. et al. (2011). Phylogenetic study of the species within the family Streptomycetaceae. Antonie Van Leeuwenhoek. Published. 